\title{
The cancer stem cell hypothesis: a work in progress
}

\author{
Brenton Thomas Tan*, Christopher Yongchul Park*, Laurie Elizabeth Ailles and \\ Irving L Weissman
}

Department of Pathology, Stanford University School of Medicine, Stanford, CA, USA

\begin{abstract}
There is a growing body of evidence that supports the idea that malignant tumors are initiated and maintained by a population of tumor cells that share similar biologic properties to normal adult stem cells. This model, the cancer stem cell (CSC) hypothesis, is based on the observation that tumors, like adult tissues, arise from cells that exhibit the ability to self-renew as well as give rise to differentiated tissue cells. Although the concept of the CSC is not entirely new, advances made over the past two decades in our understanding of normal stem cell biology in conjunction with the recent application of these concepts to experimentally define CSCs have resulted in the identification of CSCs in several human malignancies.
\end{abstract}

Laboratory Investigation (2006) 86, 1203-1207. doi:10.1038/labinvest.3700488; published online 30 October 2006

Keywords: hematopoiesis; neoplasm; transplantation; stem cells; tumor

\section{Adult stem cells as a paradigm for cancer stem cells (CSCs)}

Normal adult stem cells are tissue-specific cells that exhibit unique biologic properties, including the ability to self-renew and to differentiate into all cell types of the tissue of origin. Functionally, cells that self-renew can undergo an unlimited number of cell divisions, and with each division, they produce at least one daughter cell that maintains this indefinite capacity for cell division. When adult stem cells divide, they can also produce progenitor cells that have finite division capacity, ultimately differentiating into the mature cell types that constitute specific tissues. Based on these properties, adult stem cells form a pool of long-lived cells that provide a continuous supply of differentiated cells to their tissue compartments, which is particularly important for homeostatic control in adult tissues that undergo continuous cellular turnover, such as epithelium and blood.

That adult stem cells give rise to cancer is an attractive hypothesis, given that the classic multistep model of carcinogenesis requires a long-lived cell in which multiple genetic hits can occur. ${ }^{1}$ In

Correspondence: Dr BT Tan, MD, PhD, Department of Pathology, Stanford University School of Medicine, 300 Pasteur Drive, Room L235, Stanford, CA 94305-5324, USA.

E-mail: btan@stanford.edu

* These authors contributed equally to this work.

Received 11 June 2006; accepted 10 September 2006; published online 30 October 2006 fact, investigators had long ago postulated that cancers arise from cells with stem cell properties or directly from tissue-specific stem cells. ${ }^{2}$ Another possibility is that the progenitors of stem cells that normally undergo limited numbers of cell divisions aberrantly acquire the capacity to self-renew, subsequently becoming the long-lived target that acquires additional genetic lesions. ${ }^{3}$

Regardless of the cell of origin, whether a normal adult stem cell or progenitor, the CSC is defined by its stem cell-like properties. Like normal adult stem cells, CSCs can divide indefinitely, giving rise to both more CSCs and progeny that ultimately differentiate into the different cell types in a tumor. By maintaining at least some of the properties of their tissue of origin, CSCs give rise to tumors that phenotypically resemble their origin, either by morphology or by expression of tissue-specific genes. However, what distinguishes cancerous tissue from normal tissue is the loss of homeostatic mechanisms that maintain normal cell numbers, and much of this regulation normally occurs at the stem cell level.

In addition to providing an elegant model for carcinogenesis, the CSC hypothesis raises several important experimental and clinical implications. First, if a population of biologically unique CSCs exists, then tumor cells lacking stem cell properties will not be able to initiate self-propagating tumors, regardless of their differentiation status or proliferative capacity. This corollary is an important consideration in experimental strategies to define CSCs, which will be discussed below. Furthermore, 
the CSC raises the clinical implication that curative therapy will require complete elimination of the CSC population. Patients who show an initial response to treatment may ultimately relapse if even a small number of CSCs survive. On the other hand, targeted therapies that eliminate the CSC population offer the potential for cure. Given this promise, it is not surprising that the CSC hypothesis has attracted so much attention in recent years.

\section{Identification of CSCs in the hematopoietic system}

Much of our knowledge of CSC biology has come from experiments in normal and malignant hematopoiesis that led to the identification of the hematopoietic stem cell (HSC) and its malignant counterpart, the leukemia stem cell (LSC). Critical change occurred in the late 1980s and early 1990s, when the distinct cell surface marker profile that would allow for prospective isolation of normal HSCs by fluorescence-activated cell sorting (FACS) became known. ${ }^{4}$ These advances led to the isolation of HSCs as well as multipotent and oligopotent progenitors that generate all mature blood cells (Figure 1a).
Before these discoveries, LSCs could only be hypothesized, but with the knowledge of the cell surface phenotypes for hematopoietic stem and progenitor cells, investigators now had the ability to isolate similar subpopulations from acute myeloid leukemia (AML). John Dick and co-workers provided the first evidence for LSCs by using FACS to prospectively isolate cells from human AML that were able to initiate leukemia in transplanted mice. $^{5,6}$ An important aspect of these studies was the use of nonobese diabetic, severe-combined immunodeficient (NOD/SCID) mice, which had been shown previously to support engraftment of normal human hematopoietic cells. Evaluating only CD34 ${ }^{+}$AMLs, they showed that the CD $34^{+}{ }^{+}$DD $38^{-}$ fraction was highly enriched for leukemia-initiating activity in transplanted recipients, while both the $\mathrm{CD} 34^{+} \mathrm{CD} 38^{+}$and $\mathrm{CD} 34^{-}$fractions did not initiate leukemia. In addition, an engrafted leukemia could be serially transplanted into secondary recipients, providing functional evidence for self-renewal. This experimental approach-xenotransplantation followed by serial transplantation-is now widely accepted as an essential criterion in defining CSCs.

Interestingly, the CD $34^{+} \mathrm{CD}^{-} 8^{-}$cell surface phenotype of LSCs is shared by immature hematopoietic precursors including HSCs, which raises the possibility that LSCs arise from HSCs (Figure 1b). a

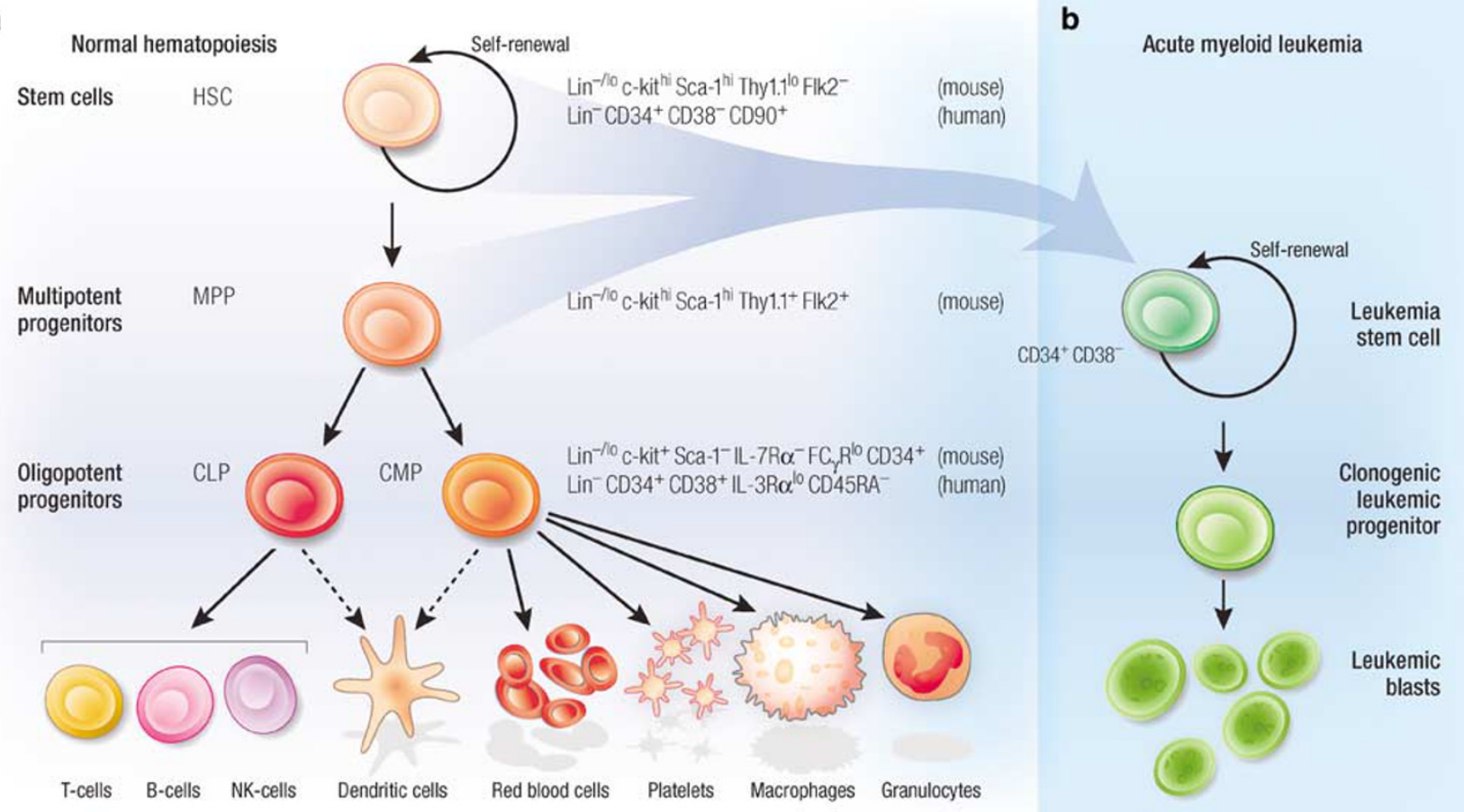

Figure 1 Normal hematopoietic stem and progenitor cells (a). The HSC is a unique hematopoietic cell by virtue of its ability to self-renew as well as generate all cells of the hematopoietic system. HSCs generate the multitude of hematopoietic cells by giving rise to MPPs and progressively lineage-restricted progenitors that have limited capacity to divide. Leukemia stem and progenitor cells (b). Similar to the HSC, the LSC has the unique ability to self-renew. LSCs give rise to clonogenic leukemic progenitors that differentiate into leukemic blasts and more differentiated progeny. Although the cell of origin of the LSC has not been elucidated, investigators hypothesize that LSCs arise from immature hematopoietic progenitors or possibly the HSC. The arrows are drawn to indicate candidate progenitor cells in which transformation occurs. 
This theory provides an attractive model for leukemogenesis since the long lifespan of the HSC allows for multiple genetic hits to occur. Additionally, based on their physiologic capacity for self-renewal, HSCs would require fewer genetic hits to become LSCs than other hematopoietic cells, which must aberrantly acquire self-renewal capacity. However, in similar studies using FACS to purify NOD/SCIDengrafting activity, Blair and co-workers showed that LSCs do not express either Thy-1 or c-kit, both of which are surface markers present on human HSCs. ${ }^{7,8}$ One possible explanation for these findings may be that transformation events result in the loss of Thy-1 and c-kit. Alternatively, final transformation may not occur in HSCs, but some other immature hematopoietic cell such as a progenitor cell that has aberrantly acquired the ability to selfrenew.

Regardless of the nature of the LSC, there is evidence that at least some genetic hits occur in HSCs. For example, the balanced reciprocal translocation $t(8 ; 21)$ is one of the most common recurrent cytogenetic anomalies in AML, resulting in the AML1-ETO fusion transcript that is present not only in leukemic blasts but also in normal hematopoietic cells and HSCs from patients in remission. ${ }^{9}$ HSCs isolated from these remission patients show normal function in vitro, maturing into normal myeloid and erythroid colonies. Taken together, these data suggest that while HSCs can acquire genetic alterations such as $t(8 ; 21)$, such events are necessary, but not sufficient for transformation. Whether final transformation occurs in a subset of HSCs with $\mathrm{t}(8 ; 21)$ or more committed progenitors remains to be resolved.

\section{Solid tumor CSCs}

While the body of work on LSCs is the most mature with respect to CSCs, investigators have recently used similar techniques to identify stem cells from solid tumors. The first such report was from Al-Hajj et $a l,{ }^{10}$ who prospectively isolated tumorigenic cells from human breast carcinoma by using FACS to purify populations able to form tumors in immunodeficient mice. Human breast carcinomas were fractionated based on their expression of CD44 and CD24, two markers shown to be heterogeneously expressed among tumor cells. Fractionated samples were implanted into the mammary pads of NOD/ SCID mice, and only the $\mathrm{CD} 44^{+} \mathrm{CD} 24^{-}$fraction contained tumor-initiating activity, whereas 100fold more cells from the CD44 ${ }^{+} \mathrm{CD} 24^{+}$or $\mathrm{CD} 44^{-}$ fractions did not form tumors. Engrafted tumors exhibited similar morphologic and immunophenotypic heterogeneity to the original specimen, containing CD $44^{+} \mathrm{CD} 24^{-}$cells as well as $\mathrm{CD} 44^{+} \mathrm{CD} 24^{+}$ and CD $44^{-}$cells. Finally, engrafted tumors could be serially transplanted, providing rigorous proof for self-renewal.
Although the work of Al-Hajj et al provides strong evidence for a breast CSC, the relationship between normal and malignant breast stem cells remains unclear (Figure 2a and b). Recently, two groups isolated candidate mouse mammary stem cells. Shackleton et $a l^{11}$ showed that cells able to reconstitute multiple breast lineages, termed mammaryrepopulating units (MRUs), are found within the $\mathrm{CD} 29^{\mathrm{hi}} \mathrm{CD} 24^{+}$fraction. Similarly, Stingl et $a l^{12}$ found that MRUs have a $\mathrm{CD} 49 \mathrm{f}^{+} \mathrm{CD} 24^{+}$Sca- $1^{\text {low }}$ immunophenotype. Although CD24 was not observed on human breast CSCs, it is unknown whether CD24 defines the human, in addition to the murine mammary stem cell. Also, further studies are needed to determine if CD44 is present on normal mammary stem cells and whether CD29, CD49f and Sca-1 define breast CSCs. Nevertheless, CD44 is expressed on the basal layer of a variety of epithelia, consistent with breast cancer originating from a less differentiated precursor.

Since the work of Al-Hajj et al, investigation of solid tumor stem cells has gained momentum, particularly in the area of brain tumors. Using in vitro culture conditions similar to those used for growth of normal neural stem cells as neurospheres, two groups independently isolated an immature, stem-cell-like population from human pediatric brain tumors. Hemmati et $a l^{13}$ and Singh et al both showed that neurosphere-forming cells express CD133, ${ }^{14}$ a cell surface marker previously shown to be expressed on normal human neural stem cells. ${ }^{15}$ Singh et $a l^{16}$ later provided more definitive proof for a stem cell population, using antibody-coated magnetic beads to enrich for populations of cells that could form tumors in the brains of NOD/SCID mice. Tumorigenic cells were contained entirely within the $\mathrm{CD}_{133^{+}}$fraction, and transplanted $\mathrm{CD}_{133^{+}}$cells gave rise to tumors with both $\mathrm{CD}_{133^{+}}$and $\mathrm{CD}^{-} 3^{-}$cells, recapitulating the original tumor cell heterogeneity. Finally, CD133+ cells could be serially transplanted, providing definite proof of self-renewal.

\section{Caveats}

While candidate CSCs have been identified for several tumor types using xenotransplantation models to demonstrate the presence of a self-renewing population, several concerns remain regarding this experimental strategy. Critics point out that xenotransplantation assays may introduce a selection bias, as engraftment may depend on other properties of cancer cells, including homing, evasion of host immune response and proliferative capacity. In addition, these xenograft experiments may not adequately model the interaction between tumor cells and the tumor microenvironment that occurs in humans, as highly purified, FACS-isolated tumor cells are used for transplantation. While such criticisms are not easily ignored, xenotransplanta- 
a Normal breast (mouse)

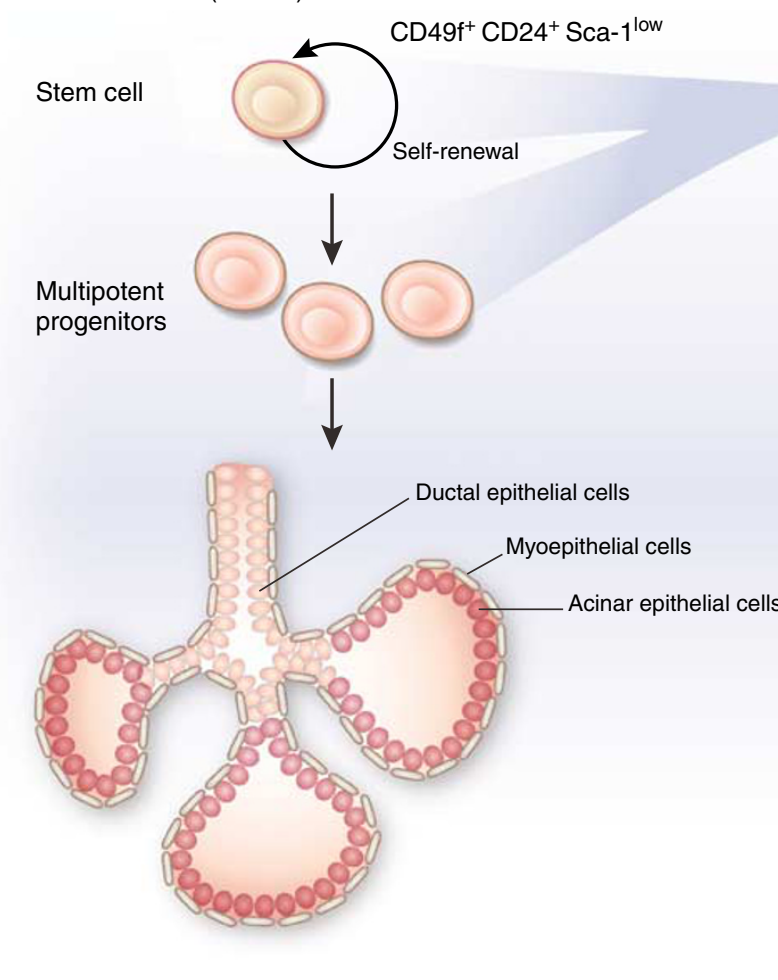

b

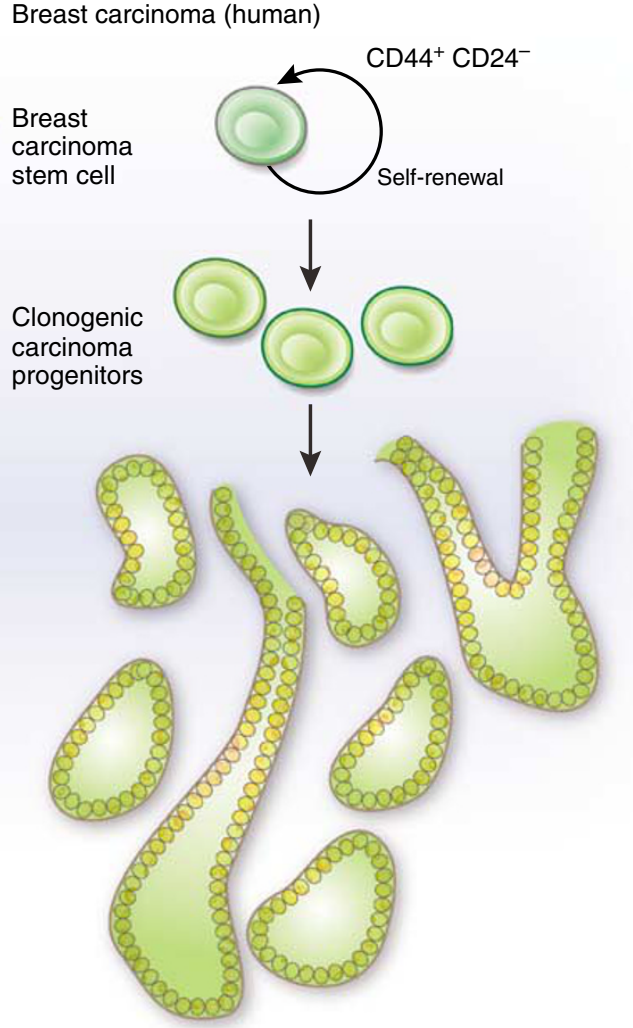

Figure 2 Proposed models for normal (a) and malignant (b) breast stem cells. Normal and malignant breast stem cells self-renew as well as give rise to progenitors with limited potential for cell division. Normal breast stem and progenitor cells are multipotent, giving rise to ductal epithelial, acinar epithelial and myoepthelial cells. In contrast, cancer stem and progenitor cells only generate carcinoma (epithelial) cells. Currently, the relationship between breast carcinoma stem cells and normal breast stem and progenitor cells remains speculative, and the arrows indicate possible cells in which transformation is postulated to occur.

tion is currently considered the most physiologically relevant model for human malignancies. Once additional insights into the biology of candidate CSCs become apparent with further characterization of their gene and protein expression profiles and validation that these molecules play a role in tumor behavior and clinical responses, it will become clear whether engrafting activity is an accurate reflection of stem cell activity.

\section{Clinical implications of CSCs}

Despite the recent surge of interest in CSCs, the clinical significance of this population remains unclear. One prediction of the CSC model is that clinical behavior should be largely dependent on the CSC population, either in quantitative terms such as the relative or absolute number of CSCs, or qualitative aspects related to biologic features of CSCs. To date, there is little data addressing this question, although a recent study suggests that in AML, a higher percentage of blasts with the $\mathrm{CD} 34^{+} \mathrm{CD} 38^{-}$ LSC phenotype is correlated with poorer overall survival. ${ }^{17}$ Clearly, more studies are needed to determine the prognostic significance of CSCs, and these data will be important in demonstrating that this experimentally defined population is relevant in humans.

In addition to prognosis, the CSC hypothesis has profound implications for cancer treatment strategies. With growing evidence that cancers arise from stem cells, both researchers and clinical investigators will increasingly focus their efforts on CSCs rather than studying whole tumors. The potential for CSC-specific therapy is currently an active area of investigation, and a recent study using a mouse AML model suggests that LSCs may be selectively targeted without adverse effects on normal HSCs. ${ }^{18}$ Interestingly, normal HSCs express high levels of drug efflux transporters, and defining whether these transporters are present in leukemic or other CSCs will be a critical step in understanding the mechanisms of tumor drug resistance. Clearly, targeting CSCs offers the potential for improvements in therapy, and future studies are likely to include the identification of CSC-specific surface markers for antibody therapy, elucidation of CSC-specific pathways that can be pharmacologically targeted and evaluation of agents that promote the differentiation of CSCs into progenitors that do not selfrenew. 
While the CSC hypothesis has exciting clinical implications, its widespread acceptance and application to the practice of medicine has yet to occur. We anticipate that in the upcoming years, CSCs will be identified in additional tumor types, and knowledge of the detailed biology and clinical significance of this experimentally defined population will provide further support for the CSC hypothesis. Ultimately, focusing research efforts on the CSC may drive important advances in our understanding of cancer biology and developing potential cures for these devastating diseases.

\section{References}

1 Vogelstein B, Fearon ER, Hamilton SR, et al. Genetic alterations during colorectal-tumor development. N Engl J Med 1988;319:525-532.

2 Cohnheim J. Ueber entzundung und eiterung. Path Anat Physiol Klin Med 1867;40:1-79.

3 Passegue E, Jamieson CH, Ailles LE, et al. Normal and leukemic hematopoiesis: are leukemias a stem cell disorder or a reacquisition of stem cell characteristics? Proc Natl Acad Sci USA 2003;100(Suppl 1):1184211849.

4 Spangrude GJ, Heimfeld S, Weissman IL. Purification and characterization of mouse hematopoietic stem cells. Science 1988;241:58-62.

5 Lapidot T, Sirard C, Vormoor J, et al. A cell initiating human acute myeloid leukaemia after transplantation into SCID mice. Nature 1994;367:645-648.

6 Bonnet D, Dick JE. Human acute myeloid leukemia is organized as a hierarchy that originates from a primitive hematopoietic cell. Nat Med 1997;3:730-737.

7 Blair A, Hogge DE, Ailles LE, et al. Lack of expression of Thy-1 (CD90) on acute myeloid leukemia cells with long-term proliferative ability in vitro and in vivo. Blood 1997;89:3104-3112.

8 Blair A, Sutherland HJ. Primitive acute myeloid leukemia cells with long-term proliferative ability in vitro and in vivo lack surface expression of c-kit (CD117). Exp Hematol 2000;28:660-671.

9 Yuan Y, Zhou L, Miyamoto T, et al. AML1-ETO expression is directly involved in the development of acute myeloid leukemia in the presence of additional mutations. Proc Natl Acad Sci USA 2001;28:1039810403.

10 Al-Hajj M, Wicha MS, Benito-Hernandez A. Prospective identification of tumorigenic breast cancer cells. Proc Natl Acad Sci USA 2003;100:3983-3988.

11 Shackleton M, Vaillant F, Simpson KJ, et al. Generation of a functional mammary gland from a single stem cell. Nature 2006;439:84-88.

12 Stingl J, Eirew P, Ricketson I, et al. Purification and unique properties of mammary epithelial stem cells. Nature 2006;439:993-997.

13 Hemmati HD, Nakano I, Lazareff JA, et al. Cancerous stem cells can arise from pediatric brain tumors. Proc Natl Acad Sci USA 2003;100:15178-15183.

14 Singh SK, Clarke ID, Terasaki M, et al. Identification of a cancer stem cell in human brain tumors. Cancer Res 2003;63:5821-5828.

15 Uchida N, Buck DW, He D, et al. Direct isolation of human central nervous system stem cells. Proc Natl Acad Sci USA 2000;97:14720-14725.

16 Singh SK, Hawkins C, Clarke ID, et al. Identification of human brain tumour initiating cells. Nature 2004;432:396-401.

17 van Rhenen A, Feller N, Kelder A, et al. High stem cell frequency in acute myeloid leukemia at diagnosis predicts high minimal residual disease and poor survival. Clin Cancer Res 2005;11:6520-6527.

18 Yilmaz OH, Valdez R, Theisen BK, et al. Pten dependence distinguishes haematopoietic stem cells from leukaemia-initiating cells. Nature 2006;441:475-482. 